\title{
La « question musulmane » en France au prisme des sciences sociales
}

Le savant, l'expert et le politique

The "Muslim Issue" in France Through the Prism of Social Sciences. The

Scholars, the Experts and the Politicians

\section{Vincent Geisser}

\section{OpenEdition}

Journals

Édition électronique

URL : http://journals.openedition.org/etudesafricaines/17041

DOI : 10.4000/etudesafricaines. 17041

ISSN : $1777-5353$

Éditeur

Éditions de l'EHESS

\section{Édition imprimée}

Date de publication : 1 juin 2012

Pagination : 351-366

ISBN : 978-2-7132-2349-5

ISSN : 0008-0055

\section{Référence électronique}

Vincent Geisser, «La « question musulmane » en France au prisme des sciences sociales », Cahiers d'études africaines [En ligne], 206-207 | 2012, mis en ligne le 01 juillet 2014, consulté le 20 avril 2019 URL : http://journals.openedition.org/etudesafricaines/17041; DOI : 10.4000/etudesafricaines. 17041

Ce document a été généré automatiquement le 20 avril 2019

(c) Cahiers d'Études africaines 


\title{
La « question musulmane » en France au prisme des sciences sociales
}

\author{
Le savant, l'expert et le politique \\ The "Muslim Issue" in France Through the Prism of Social Sciences. The \\ Scholars, the Experts and the Politicians
}

Vincent Geisser

\section{Un objet « chaud », source de luttes symboliques}

1 Longtemps discrète dans le champ des sciences sociales, la «question musulmane » en France connaît depuis la fin des années 1980 un regain de visibilité, dont le signe le plus probant est sans doute le foisonnement d'articles, d'ouvrages, de rapports d'expertise et de prises de parole médiatiques, dont le caractère académique soulève de nombreuses interrogations. Cette ambivalence ne ressort pas tant de l'absence de rigueur de leurs auteurs, qui sont souvent des chercheurs et des universitaires confirmés appartenant à des institutions académiques reconnues (CNRS, EHESS, EPHE, Science Po, etc.) $)^{1}$ que du statut de l'objet lui-même à la confluence de demandes multiples. En effet, la «question musulmane » en France constitue un " objet chaud», dont la construction est source de débats vifs entre des acteurs aux intérêts contradictoires, sinon divergents. La réflexion critique du politologue Jean Leca (1988: 43-44), développée il y a plus de vingt ans, paraît toujours pertinente aujourd'hui :

«Le chercheur est dès lors doublement perturbé, par l'objet lui-même et par sa propre participation, inévitable malgré qu'il puisse (quelquefois) en avoir, aux "bruits" émis pour nommer cet objet. Il ne peut imposer un "silence dans les rangs" de cet ensemble si rebelle où, pour de très bonnes raisons sociologiques, la lutte idéologique n'a à peu près rien à avoir avec un échange réglé d'arguments logiques et vérifiables à l'intérieur d'un cadre argumentatif commun puisque l'objet de la lutte n'est pas seulement, comme le répète à l'envie la science politique, la "mise sur" mais l'imposition de ce cadre argumentatif lui-même. » 
2 En ce sens, étudier la « question musulmane » en France revient autant à analyser l'objet que les luttes symboliques - mais bien réelles - qui en définissent les contours et en déterminent la saillance dans le débat public.

3 De ce point de vue, l'hégémonie du discours de la science politique sur l'islam en France ne tient probablement pas au hasard: les politologues apparaissaient, en effet, les mieux placés pour répondre aux sollicitations politiques et institutionnelles en matière de connaissance des populations musulmanes résidant sur le territoire français. Leur proximité avec le champ politique les conduit à être les interlocuteurs «naturels » des décideurs en la matière, les transformant parfois en « éclaireurs ", voire en conseillers du Prince (Achcar 2008). Ainsi, les approches politologiques relatives à « l'islam transplanté » (celui des immigrés et de leurs descendants) épousent indirectement les préoccupations politiques du moment, tout en conservant une certaine neutralité axiologique qui vient attester, malgré tout, de leur scientificité (Dassetto 1994 : 201-211). À quelques rares exceptions près, les politologues versent rarement dans l'expertise ministérielle, même si leurs écrits de vulgarisation présentent des accointances évidentes avec la logique des experts. À l'inverse, les anthropologues des religions et les sociologues des migrations se sont longtemps désintéressés de la question de l'islam en France : les premiers, parce qu'ils se sont davantage concentrés sur les «acteurs musulmans » des sociétés d'origine (Maghreb, Moyen-Orient et Afrique noire), les considérant comme les dépositaires légitimes de traditions, de pratiques et de croyances ancestrales, alors que le phénomène musulman en immigration leur apparaissait comme une version affadie ou déviante d'une culture autochtone plus "authentique "; les seconds, parce qu'ils avaient tendance à traiter la question religieuse comme une dimension négligeable ou résiduelle du processus d'intégration, privilégiant davantage les aspects économiques et sociaux (école, délinquance, identité, vie dans les quartiers, etc.) (ibid.). Aussi, anthropologues et sociologues ont-ils eu du mal à considérer l'islam transplanté comme un «fait social ordinaire ", le cantonnant à un statut d'exceptionnalité, lié à la conjoncture politique.

4 Ce désintérêt des anthropologues et des sociologues pour la vie religieuse des musulmans de France - même si cela change aujourd'hui avec l'émergence d'une nouvelle génération de chercheurs - permet de comprendre, en partie, cette difficulté de l'objet «islam transplanté » à se fondre dans les logiques du champ scientifique qui suppose une certaine distance par rapport aux émotions et aux passions agissantes. Mais elle n'explique pas tout. Car, si l'islam de France reste un « objet chaud » et donc difficilement conceptualisable aux yeux des scientifiques, cela tient aussi très largement à son mode de construction historique qui relève de la "zone grise ", à savoir des relations ambivalentes entre savants et politiques. Sur ce plan, les orientalistes français du $\mathrm{XIX}^{\mathrm{e}}$ et $\mathrm{XX}^{\mathrm{e}}$ siècles, qui n'en étaient pas moins érudits et dont les objets d'étude s'inscrivaient pleinement dans le champ académique de l'époque (légitimation universitaire, publications scientifiques nombreuses et reconnaissance institutionnelle par leurs pairs), ont été les premiers à entretenir des relations de proximité avec le champ politique, répondant aux demandes liées aux impératifs de la conquête coloniale et à la nécessité de l'administration de comprendre des populations majoritairement musulmanes (Berbères, Arabes, Touaregs, Swahilis, etc.) (Luizard 2006). Très tôt, la politique musulmane de la France a donc fait appel aux lumières des savants, faisant dire à l'historien Henry Laurens (2004b : 257) " Point de politique musulmane sans science sociale du monde musulman », signifiant par là les liens historiques très forts entre la production scientifique sur l'islam et la gestion politique des dits « musulmans ». 


\section{Islamologie et pouvoir : des relations anciennes et ambivalentes}

Il serait exagéré de faire porter la responsabilité de l'entreprise coloniale aux orientalistes européens. D'une part, parce que ces derniers étaient souvent traversés par des débats contradictoires et des perceptions $\mathrm{du}$ " monde musulman » très hétéroclites, loin de constituer un «bloc » homogène de savants au service de la colonisation (ibid. 103-128). Une lecture simpliste de l'œuvre-phare d'Edward Saïd (1980) conduit parfois à une telle caricature, comme si l'orientalisme universitaire avait constitué le «bras intellectuel » de la domination coloniale des peuples musulmans (Marzouki 2004 : 62-67). D'autre part, parce que l'orientalisme a moins entretenu une relation de collaboration active avec les politiques qu'une convergence de vues, renvoyant à « l'air du temps » et à l'idéologie ambiante que l'on pourrait ramener à la notion de «messianisme civilisateur ». Il est vrai, toutefois, que les relations entre les savants orientalistes et les politiques du moment étaient marquées en permanence par l'ambivalence, incitant certains auteurs actuels à parler d'« islamologie officielle », consacrant l'alliance entre les milieux académiques et l'administration coloniale :

«L'islamologie officielle ne s'embarrassait pas de scrupules humanitaires et n'hésitait pas à se mettre au service des hommes d'État et des militaires. La politique coloniale imposait, en effet, en ce qui concerne l'islam, entre la science et l'État une relation particulière, comparable aux liens entre politique et religion dans un système clérical réfractaire à la laïcité ».(Sellam $2006: 46)$

Dès le début $\mathrm{du} \mathrm{xx}^{\mathrm{e}}$ siècle, des grandes figures de l'orientalisme français (Alfred Le Chatelier, Louis Massignon, Louis Gardet, Robert Montagne, etc.) vont ainsi siéger dans des commissions et des institutions d'État, dont la mission première est de fournir à l'administration coloniale les ressources intellectuelles lui permettant de mieux gérer les populations musulmanes nouvellement conquises. Qu'il s'agisse de la Commission interministérielle des affaires musulmanes (CIAM, créée en 1911), du Haut comité méditerranéen (нсм, 1936) ou du Centre des hautes études sur l'Afrique et l'Asie modernes (CHEAM, 1937) ${ }^{2}$, à chaque fois les savants orientalistes sont sollicités pour éclairer les politiques et les administrateurs sur des aspects généraux relatifs aux peuples colonisés (traditions, mœurs, cultures, histoire, etc.), mais aussi, plus spécifiquement, sur la question religieuse: l'islam est déjà perçu à l'époque comme l'un des principaux problèmes (au sens de "problème public »), en raison de sa capacité à rassembler et à susciter un mouvement d'identification et de contestation contre l'entreprise coloniale : «Il s'agit de donner à la politique musulmane un point d'appui solide, "la meilleure de nos forces intellectuelles, l'Université" » (Laurens 2004b : 258).

7 Cependant, cette présence effective des savants au sein d'instances directement liées à la politique coloniale de la France ne signifie pas forcément collaboration aveugle et légitimation totale des décisions gouvernementales. Au contraire, l'étude des procèsverbaux des débats révèle des points de vue contrastés, des divergences, voire des frictions, certains savants, tels que Louis Massignon, n'hésitant pas à faire valoir une vision à la fois libérale et humaniste de l'administration des populations, refusaient de cautionner certaines orientations clairement « antimusulmanes » des autorités publiques (ibid. ; Sellam 2006).

8 Loin de se limiter aux territoires colonisés du Maghreb, d'Afrique noire et du ProcheOrient, le savoir des islamologues va connaître également des sollicitations politiques 
dans le cadre métropolitain, préfigurant ainsi la naissance d'un nouvel objet de recherche davantage lié aux enjeux internes à l'espace hexagonal :

«Avec les premières migrations massives, dès le début du siècle, mais surtout à partir de la première guerre mondiale, cette philosophie et ces représentations vont accompagner les migrants en métropole. Le processus n'est pourtant possible, tel qu'il s'est déroulé, que parce que de la colonie à la métropole, se transfèrent en même temps que la main-d'œuvre ou les soldats coloniaux, des spécialistes, un savoir-faire et une science de ce qui est appelé "le sujet indigène musulman originaire de nos possessions en Afrique du Nord" ».(Abdelfettah 2004)

C'est l'acte de naissance d'une islamologie française appliquée aux musulmans résidant dans l'hexagone, bien que cet épisode soit largement méconnu par les spécialistes actuels. Et Henry Laurens (2004b : 279) a probablement raison de rappeler que la notion d'« islam français » ne doit rien aux politiques et aux politologues d'aujourd'hui mais qu'elle était déjà en usage au début du siècle dernier :

"Durant la Grande Guerre, le terme d'“islam français" devient d'un usage courant à la fois en raison de l'enjeu musulman global et de la première arrivée massive des musulmans, soldats ou ouvriers, en métropole. »

10 La «question musulmane » en France aurait donc pu devenir logiquement l'un des objets d'étude privilégié des islamologues et cela d'autant que le nombre de "musulmans » résidant sur le territoire national et dans l'espace européen ne cessait de croître d'année en année, favorisant un processus d'institutionnalisation du fait islamique à travers les associations, les cercles intellectuels et les personnalités religieuses. Pourtant, force est de constater que cette «islamologie hexagonale » ne fut qu'embryonnaire et qu'elle ne connut qu'un faible développement au sein de l'université française, à tel point que les islamologues d'aujourd'hui ne s'intéressent guère à l'islam de France qu'ils tendent à traiter comme un objet trivial, peu digne d'intérêt scientifique. L'islam des immigrés leur apparaitt majoritairement comme un « islam dévoyé », un « islam déraciné » qui ne mérite sans doute pas que l'on s'y intéresse dans le cadre des cursus classiques d'islamologie et des études arabisantes. Comme le fait remarquer le sociologue Félice Dassetto (1994: 2002), l'islamologie classique a très largement raté le coche de la naissance d'un islam européen, dont les expressions, certes récentes, n'en sont pas moins pertinentes et stimulantes pour l'observateur scientifique :

« Du point de vue méthodologique, les orientalistes et les islamologues arrivèrent rapidement à la limite de leurs possibilités: ils pouvaient expliquer l'islam "théorique" et l'islam dans les textes, mais ils ne disposaient pas de méthodes et de concepts pour une analyse de l'islam vécu et pratiqué. En outre, ils tendaient souvent à se transformer d'analystes en acteurs qui entendaient dire aux musulmans européens, porteurs d'un islam populaire, la nature de l'islam "vrai", purifié des scories des traditions non islamiques: en réalité de l'islam qu'euxmêmes percevaient comme vrai. »

11 Le désengagement de l'islamologie classique des problèmes du temps présent s'explique aussi très largement par le processus de "décolonisation mentale » qui a touché les sciences sociales et le discrédit idéologique jeté sur la tradition orientaliste qui a été contrainte de se replier sur le milieu universitaire et sur les études classiques, créant un vide dans la compréhension des phénomènes islamiques contemporains :

«[La] critique de l'orientalisme traditionnelle a permis le développement de la science politique, qui en mettant en exergue de nouvelles méthodes sur l'analyse du monde arabo-musulman a également provoqué ce déni d'histoire, car la réflexion porte sur une globalisation des phénomènes et un rétrécissement au très contemporain ».(Bouissa 2006) 
12 être l'œuvre de Jacques Berque : orientaliste, arabisant, fin connaisseur du Maghreb et du Machrek, traducteur du Coran, il n'en développa pas moins un intérêt certain pour l'islam transplanté, porté par les immigrés et leurs descendants. À ce titre, il participa à plusieurs consultations et rapports officiels sur les questions d'immigration, d'intégration et d'islam dans les sociétés d'accueil (Lorcerie 1998 : 171-194), alors que la majorité de ses collègues orientalistes préféraient se cantonner au champ universitaire. Mais les apports intellectuels de Jacques Berque (1989), sa capacité à conceptualiser l'islam « des deux rives » et à analyser la dimension "islamo-méditerranéenne » de l'Europe ne furent jamais reconnus à leur juste valeur dans le monde universitaire et encore moins par les institutions publiques où il ne bénéficiait que de très rares soutiens ${ }^{3}$. La disparition de Jacques Berque en 1995 symbolise à elle seule la difficulté de l'islamologie classique à s'emparer de l'islam européen comme objet scientifique (Benkirane 2003) et surtout l'émergence d'un nouveau courant de recherche, celui des politologues, qui contribuera à promouvoir une «néo-islamologie », certes en phase avec son temps mais pauvre pour ce qui est des références historiques et théoriques.

\section{La néo-islamologie des politologues : entre recherche et expertise}

L'effacement des islamologues classiques sur le terrain des phénomènes musulmans du temps présent est concomitant de la montée en puissance des politologues qui, à l'horizon des années 1980, conquièrent une position quasi hégémonique sur la question. En effet, une consultation des bases bibliographiques révèle que la très grande majorité des travaux de recherche universitaires sur l'islam de France est le produit d'institutions fortement liées à la science politique ou à la sociologie du politique, entretenant généralement des relations de proximité avec les organismes officiels liés à l'État. Ce n'est donc pas un hasard si les instituts d'études politiques - notamment celui de Paris et d'Aix-en-Provence - , autour de professeurs en science politique comme Rémy Leveau ${ }^{4}$ et Bruno Étienne ${ }^{5}$ seront, dès le milieu des années 1980, les principaux lieux de production et de diffusion d'un nouveau savoir sur l'islam de France, marqué par le souci de coller à la demande institutionnelle et reflétant, en partie, les préoccupations intégratrices et sécuritaires des pouvoirs publics. Les politologues sont sollicités par les autorités publiques afin de les aider à définir une "nouvelle politique musulmane ", soucieuse d'intégrer les éléments modérés de la communauté musulmane, tout en prévenant les dérives extrémistes :

«[...] Les politologues, et dans une moindre mesure les juristes, furent sensibles, en raison de leur proximité institutionnelle et méthodologique, à la demande du politique d'inclure cette réalité nouvelle, perçue comme mouvante et insaisissable, dans la réalité de l'État ».(Dassetto $1994: 202$ )

14 Ce prisme politologique, davantage prégnant en France que dans les autres pays européens, probablement en raison de la présence d'un certain «jacobinisme scientifique ", aura des conséquences directes sur les modes opératoires des collectes des données (enquêtes et investigations) et sur les manières de conceptualiser l'objet « islam » :

«En France en particulier la question générale de la politisation de l'islam a été largement traitée. Importantes sont les publications relatives à l'insertion de l'islam dans les espaces institutionnels et juridiques. Le développement de ces analyses 
confirme la sensibilité des scientifiques à l'égard des questions que se posent l'opinion publique ou les pouvoirs à l'égard des musulmans ».(ibid. : 204) politologiques sur l'islam de France (fort écho dans les champs médiatique et politique), il est à noter également leur tendance récurrente à relier la "question religieuse » au «problème des banlieues». La publication de l'ouvrage de Gilles Kepel (1987)6, Les banlieues de l'islam. Naissance d'une religion en France, est emblématique de cette évolution où les nouveaux modes de mobilisation des acteurs islamiques dans l'hexagone sont systématiquement reliés au «mal vivre» des quartiers populaires, expliquant entre autres que les descendants de migrants nord-africains (les « Beurs ») chercheraient dans la religion une nouvelle voie de salut. De telles recherches vont alors accréditer le thème d'un «retour du religieux» dans les nouvelles générations socialisées en France, négligeant les modes de socialisation et de transmission familiales qui jouent pourtant un rôle majeur. C'est l'idée que l'islamisation des banlieues serait le fait d'une " génération spontanée ", acculturée et tiraillée entre deux cultures, l'islam leur offrant une " nouvelle identité ", distincte à la fois de la culture traditionnelle héritée de leurs parents et de la culture dominante ("catho-laïque ») inculquée par la société d'accueil. Félice Dassetto (1994 : 205) a raison d'interroger la pertinence de la thèse du « retour du religieux » qui a parfois tendance à évacuer les sociabilités ordinaires au profit des explications politiques liées notamment à l'activisme et au prosélytisme de certains groupes islamiques, comme si cet «islam des jeunes » venait de nulle part ou plutôt qu'il leur était imposé de l'extérieur :

«Peu de travaux ont jusqu'à présent développé l'analyse des processus de socialisation à l'islam, ce qui reviendrait à analyser les diverses formes de transmission religieuse à travers les générations (école coranique, socialisation familiale, enseignement de l'islam, socialisation quotidienne), la prédication islamique, la socialisation confrérique et organisationnelle et les multiples médias (cassettes et vidéo, livres et revues), la circulation commerciale d'une ampleur frappante. »

16 À la différence des autres religions, le discours scientifique sur l'islam du temps présent emprunte un mode dramatique, même si, au final, les politologues viennent rassurer leurs lecteurs sur la «normalité » et la non-dangerosité de leurs enquêtés musulmans. On saisit là toute la difficulté éprouvée par les politologues, ces vingt dernières années, à s'extraire intellectuellement du débat politico-médiatique et à esquisser des problématiques de recherche véritablement originales et autonomes par rapport à la demande institutionnelle du moment. Du coup, la néo-islamologie des politologues, alors qu'elle se fonde généralement sur des enquêtes sérieuses et des démarches rigoureuses sur le plan scientifique, donne souvent l'impression de "cousiner" avec une forme d'expertise médiatique et politique "améliorée ", comme le relevait très justement l'historien africaniste Jean-Louis Triaud (1998: 18) : « Le temps médiatique impose l'étude sur le court terme, c'est une islamologie qui se fait sismologie. »

Il faut reconnaître toutefois que les politologues et certains sociologues de l'École des hautes études en sciences sociales ${ }^{7}$ ont été les pionniers des travaux sur le fait musulman hexagonal dans les années 1980, contribuant à former une nouvelle génération d'enseignants-chercheurs (Valérie Amiraux, Florence Bergeaud-Blacker, Amel Boubakeur, Jocelyne Césari, Franck Frégosi, Vincent Geisser, Nacira Guénif, Khadija Mohsen-Finan, Nadine Weibel et bien d'autres) davantage en phase avec les réalités empiriques européennes et moins dépendants de la demande politique. Avec le temps, on peut

Cahiers d'études africaines, 206-207 | 2012 
constater que les objets de recherche traitant de l'islam de France deviennent de plus en plus « ordinaires", se détachant du seul prisme politique pour s'inscrire davantage dans les problématiques des sciences sociales "classiques». Les doctorants et les jeunes docteurs des années 2000 investissent de nouveaux sujets et des terrains plus " sociologiques ", voire anthropologiques, moins marqués par les registres institutionnels et sécuritaires, comme les consommations hallal, l'islam au féminin, les mobilisations musulmanes en milieu étudiant, les réseaux confrériques, les modes de croyance, les pratiques religieuses privées et publiques, la question du culte musulman, etc. ${ }^{8}$. Désormais, le fait musulman se banalise, traité par les sciences sociales en objet de recherche "comme les autres", remettant en cause son statut d'exception même si les approches institutionnelles (rapport entre les musulmans et les pouvoirs publics) sont encore très présentes et même si certains anthropologues et sociologues spécialistes des religions (christianisme, judaïsme, etc.) persistent parfois à voir dans l'islam une « religion étrange » (Césari 1997-1998 : 41-58).

Si certains esprits critiques (Achcar 2008) sont tentés de clouer au pilori les politologues pour avoir préparé le terrain à l'expertise sécuritaire en matière d'islam, il convient de préciser qu'ils ne se sont jamais confondus avec elle, parvenant à maintenir une certaine marge d'autonomie. Pour preuve, au lendemain des attentats du 11 septembre 2001, les spécialistes de science politique seront les premiers sur le banc des accusés, montrés du doigt par les tenants du discours sécuritaire, leur reprochant leur aveuglement scientifique et leur angélisme irénique.

\section{L'Après-11 septembre 2001 : triomphe des experts sécuritaires et marginalisation de la parole des chercheurs}

19 Les attentats du 11 septembre auront un effet inattendu sur la « petite communauté » des politologues français spécialistes du fait musulman. Ces derniers seront l'objet d'une forme de procès en sorcellerie mené par une certaine presse et par les experts sécuritaires qui leur reprocheront leur trop grande bienveillance à l'égard des «milieux musulmans ». On peut lire ainsi dans un hebdomadaire français à grand tirage sous la plume de François Dufay (2001) :

« En pulvérisant les tours jumelles du World Trade Center, les fous d'Allah n'ont pas seulement ridiculisé la CIA et le FBI. Ils ont aussi pris à revers la très docte confrérie des spécialistes français de l'islam [...]. Il reste que bien peu d'experts ont anticipé l'actuelle déflagration de haine religieuse. Et la question ne peut être esquivée : les islamologues n'ont-ils pas eu tendance, par angélisme ou par souci du "politiquement correct", à minorer la menace que font peser sur le monde Ben Laden et consorts? À lire certains ouvrages, les fanatiques de la charia apparaissent plus comme des adeptes d'une sorte de théologie de la libération que d'un totalitarisme enturbanné [...]. La peur de l'islam ? Des "caricatures", des "fantasmes anxiogènes" de journalistes occidentaux [...]. En réalité, aux yeux d'islamologues aussi éminents que Bruno Étienne, François Burgat ou Jocelyne Césari, l'Occident, quoi qu'il fasse, sera toujours plus coupable que l'Orient, Israël que les Palestiniens, les colonels algériens que le GIA... ».

En somme, les politologues français auraient pêché par islamophilie militante, développant une véritable empathie avec leur objet de recherche (les musulmans), leur empêchant d'anticiper les dérives radicales. S'il est vrai, qu'au départ, ce procès en sorcellerie ne s'adresse qu'à une minorité de politologues, il touche très vite l'ensemble de la profession et des spécialistes du fait musulman : ces derniers se voient de plus en 
plus marginalisés des appels d'offre institutionnels et leur parole publique se réduit comme peau de chagrin au profit d'une catégorie d'acteurs de plus en plus influente : les experts sécuritaires (Bigot, Bonelli \& Deltombe 2008; Geisser 2003). Cette hégémonie médiatique des experts et des think-tanks sécuritaires n'aurait que de peu d'importance pour notre propos, si elle ne venait pas concurrencer directement les social scientists sur leur terrain. Car, précisément, dans le cas français post-11 septembre, ces nouveaux experts sécuritaires se parent des attributs d'une certaine scientificité, contribuant à engendrer un phénomène de brouillage des frontières entre, d'une part le champ scientifique et celui de l'expertise et, d'autre part, entre l'analyse sociologique et l'essayisme. Développant une vision extrêmement superficielle des phénomènes relatifs à l'islam en France, généralement abordés sous l'angle exclusif du fondamentalisme et de l'extrémisme religieux, les experts et les essayistes se réfugient derrière l'apparente neutralité de leurs analyses pour asséner à leurs auditeurs ou à leurs lecteurs des «fausses évidences » sur les musulmans de France et d'Europe (intégrisme, djihadisme, réseaux dormants d'Al Qaïda, etc.) (Bigo, Bonelli \& Deltombe 2008). La force du discours des experts "ès islam " est d'apparaitre neutre, alors que, de manière paradoxale, le discours des chercheurs semble davantage engagé et donc soupçonnable de partialité et de compassion pour les acteurs musulmans. Du coup, le discours des experts sécuritaires revendique une supériorité heuristique sur le discours des chercheurs en sciences sociales, en mettant en avant les arguments du réalisme et de la capacité prédictive :

«Si ces discours [d'experts] sont en effet capables d'énoncer (d'annoncer ?) les problèmes - toujours graves - du siècle, ils peuvent tout aussitôt énumérer les solutions pour y faire face. De multiples indicateurs techniques sont mobilisés pour montrer que la force de l'argument repose sur du concret, du chiffre, du vrai [...] et donc de l'indiscutable. L'approche descriptive du réel est quasi clinique : diagnostic et remède, avec une seule explication possible dans la détermination des problèmes et une seule voie pour y remédier. Il ne s'agit pas de programmation politique à proprement parler car la voie à emprunter serait naturelle. Le discours expert tire, en effet, sa légitimité de sa grande capacité à pouvoir paraphraser le réel [...]». (Cussó \& Gobin 2008 : 5-11)

Toutefois, cette percée fulgurante de l'expertise et de l'essayisme sur la question de l'islam en France n'a que faiblement perturbé les logiques de fonctionnement du champ scientifique : rares sont les experts sécuritaires qui ont fait des intrusions significatives dans le champ des sciences sociales pour y imposer leur vision anxiogène des phénomènes liés à la « présence musulmane » en Europe. Au contraire, ce développement croissant de l'expertise en matière d'islam aurait même créé un effet de feed-back positif en contraignant les politologues et les sociologues à se recentrer davantage sur leurs objets d'étude et à clarifier leur position de chercheur par rapport aux multiples sollicitations médiatiques, institutionnelles et sécuritaires.

\section{Une sociologie du fait musulman libérée des prismes politique et sécuritaire?}

Dans son bilan des recherches européennes sur l'islam et les musulmans, réalisé au milieu des années 1990, le sociologue Félice Dassetto (1994) en appelait à sortir de « l'exception islamique » dans les sciences sociales pour reconsidérer ces phénomènes comme des faits sociaux ordinaires. Quinze ans après l'émission de ce "vœu scientifique », l'état des lieux apparaît plus que contrasté, en raison des ambivalences historiques persistantes qui ont accompagné le développement de cet objet au sein du champ des sciences sociales et 
humaines. Car, comme nous l'avons vu, le problème principal ne réside pas tant dans l'absence de scientificité des études ou le défaut de formation académique de leurs auteurs mais davantage dans les nombreuses transactions collusives des spécialistes de l'islam du temps présent avec les autres champs sociaux (médiatique, politique, administratif, etc.), expliquant que le fait musulman en Europe reste toujours un « objet chaud» (Leca 1988). Pour preuve, les islamologues du siècle dernier, réputés pourtant pour leur érudition encyclopédique et leur connaissance approfondie des sociétés dites «musulmanes» du Maghreb, du Machrek et d'Afrique noire, n'en entretenaient pas moins des relations ambivalentes avec les institutions politiques, jouant parfois le rôle de "conseillers" en matière de gestion des «indigènes musulmans ». Leurs "continuateurs», les néo-islamologues issus pour la plupart de la science politique, poursuivront cette relation ambivalente qui se renforcera d'ailleurs sous l'effet de la société de communication : objet chaud, l'islam deviendra également un objet médiatique et les politologues seront de plus en plus fréquemment sollicités par la presse et les entreprises audiovisuelles pour donner leur «avis» et commenter les sondages sur "l'intégration des musulmans» de France et d'Europe (Deltombe 2005). Une occasion historique a probablement été manquée au cours des années 1960-1970 avec le «repli universitaire» de l'islamologie classique qui, parce qu'elle était réticente à la vulgarisation et la trivialisation de l'objet «islam », a choisi d'ignorer délibérément les phénomènes musulmans du temps présent liés notamment aux courants migratoires et à la présence de plus en plus visible de musulmans dans l'espace européen. On peut regretter, en effet, que la troisième génération des islamologues, représentée par des figures savantes prestigieuses telles que Mohamed Arkoun, Michel Chodkiewicz, Azzedine Guellouz, Ali Mérad, Eva de Vitray-Meyerovitch, etc., n'ait pas davantage été présente dans les débats scientifiques sur l'islam et les musulmans européens. Cette absence des islamologues se fait ressentir encore aujourd'hui par une sorte de dichotomie académique entre les chercheurs arabisants issus des cursus littéraires qui travaillent principalement sur l'islam classique, et les socio-politologues qui investissent massivement les sujets et les thèmes liés aux "musulmans contemporains ». En France, ces deux «corporations universitaires » coexistent pacifiquement au sein du CNRS et de l'université, mais ne se rencontrent presque jamais, si ce n'est qu'à de rares occasions: les colloques et les publications communes relèvent de l'exception. Toutefois, l'émergence d'un «espace musulman européen ", empruntant ses références dogmatiques et théologiques autant aux sociétés musulmanes historiques qu'aux phénomènes diasporiques (islam transplanté, conversions, circulations des personnalités islamiques entre les deux rives... ), pourrait fournir de la matière scientifique et éveiller des vocations islamologiques chez les jeunes enseignants-chercheurs, à la fois soucieux de se conformer aux règles du champ scientifique, tout en contribuant à éclairer les problématiques actuelles liées à la « question musulmane » auprès des publics profane et initié. 


\section{BIBLIOGRAPHIE}

ABDELFETTAH, N.

2004 - " "Science coloniale" et modalités d'encadrement de l'immigration algérienne à Paris ", Bulletin de l'IHTP [Institut d'histoire du Temps présent], dossier « Répression, contrôle et encadrement dans le monde colonial au xx ${ }^{\mathrm{e}}$ siècle », 83, <http://www.ihtp.cnrs.fr/spip.php\% 3Farticle332\&lang=fr.html>.

ACHCAR, G.

2008 - « L'orientalisme à rebours : de certaines tendances de l'orientalisme français après 1979 », Mouvements, $54: 127-144$.

AMIRAUX, V.

2001 - Acteurs de l'islam entre Allemagne et Turquie, Paris, L'Harmattan (« Logique politique »).

ARKOUN, M.

2006 - Histoire de l'islam et des musulmans en France du Moyen Âge à nos jours, Paris, Albin Michel.

BENKIRANE, R.

2003 - « Jacques Berque. Une sociologie vaste et profonde », Postface à l'ouvrage de Jacques Berque, Quel islam ?, Paris, Sinbad-Actes Sud.

BERGAUD-BLACKER, F. \& BERNARD, B.

2010 - Comprendre le halal, Paris, Édipro.

BERQUE, J.

1989 - Mémoires des deux rives, Paris, Éditions du Seuil.

BIGO, D., BONELLI, L. \& DELTOMBE, T. (DIR.)

2008 - Au nom du 11 septembre. La démocratie à l'épreuve du terrorisme, Paris, La Découverte.

BOUBAKEUR, A.

2004 - Le voile de la mariée. Jeunes musulmanes, voile et projet matrimonial en France, Paris, L'Harmattan (« Perspectives méditerranéennes »).

BOUISSA, $\mathrm{H}$.

2006 - « Islam et historiographie : la construction d'une image », <http://oumma.com/Islam-ethistoriographie-francaise>.

CÉSARI, J.

1990 - Musulmans et républicains. Les jeunes, l'islam et la France, Bruxelles, Éditions Complexe.

1994 - Être musulman en France : associations, militants et mosquées, Paris, Karthala.

1997 - Faut-il avoir peur de l'islam ?, Paris, Presses de Sciences Po («Bibliothèque du citoyen »).

1997-1998 - « "Cette étrange étrangeté” : les représentations françaises de l'islam », Confluences Méditerranée, 24 : 41-58.

2003 - L'islam à l'épreuve de l'Occident, Paris, La Découverte.

CHODKIEWICZ, M.

1986 - Le Sceau des Saints, Prophétie et Sainteté dans la doctrine d'Ibn Arabî, Paris, Gallimard. 
CUSSó, R. \& GOBIN, C.

2008 - « Du discours politique au discours expert : le changement politique mis hors débat? ", Mots. Les langages du politique, 88 : 5-11, <www.cairn.info/revue-mots-2008-3-page-5.htm>.

DASSETTO, F.

1994 - «L'Islam transplanté : bilan des recherches européennes ", Revue européenne des migrations internationales, 10 (2) : 201-211.

1996 - La construction de l'islam européen. Approche socio-anthropologique, Paris-Montréal,

L'Harmattan.

2004 - Islams du nouveau siècle, Bruxelles, Labor (« Quartier libre »).

DELTOMBE, $\mathrm{T}$.

2005 - L'islam imaginaire. La construction médiatique de l'islamophobie, Paris, La Découverte.

DUFAY, F.

2001 - « La fausse prophétie des islamologues », Le Point, 5 octobre.

ÉTIENNE, B. (DIR.)

1990 - L'islam en France, Paris, CNRS-Éditions.

ÉTIENNE, B.

1989a - La France et l'Islam, Paris, Hachette.

1989 b - L'islamisme radical, Paris, LGF.

FRÉGOSI, F.

2000 - La formation des cadres religieux musulmans en France : approches socio-juridiques, Paris,

L'Harmattan.

2008a - Penser l'islam dans la laïcité, Paris, Fayard (« Les dieux de la cité »).

$2008 \mathrm{~b}$ - « Les nouveaux contours du champ intellectuel musulman en France », Revue des mondes musulmans et de la Méditerranée, <http://remmm.revues.org/index5553.html>.

FRÉGOSI, F. \& WILLAIME, J.-P. (DIR.)

2001 - Le religieux dans la commune. Les régulations locales du pluralisme religieux, Paris, Labor \&

Fides.

GASPARD, F. \& KHOSROKHAVAR, F.

1995 - Le foulard et la République, Paris, La Découverte.

GEISSER, $\mathrm{V}$.

2003 - La nouvelle islamophobie, Paris, La Découverte (« Sur le Vif »).

GEISSER, V. \& ZEMOURI, A.

2007 - Marianne \& Allah. Les politiques français face à la " question musulmane », Paris, La Découverte.

GÖLE, N.

1993 - Musulmanes et modernes. Voiles et civilisation en Turquie, Paris, La Découverte.

GUELLOUZ, A.

2004 - L'islam, Paris, Fayard (« Bibliothèque de culture religieuse »).

GUENIF-SOUILAMAS, N.

1999 - Des « beurettes » aux descendantes d'immigrants nord-africains, Paris, Grasset (« Partage du savoir »).

KEPEL, G.

1987 - Les banlieues de l'islam. Naissance d'une religion en France, Paris, Éditions du Seuil. 
KHOSROKHAVAR, F.

1997 - L'islam des jeunes, Paris, Flammarion.

2004 - L'islam dans les prisons, Paris, Balland (« Voix et regards »).

LAURENS, $\mathrm{H}$.

2004a - «L'orientalisme français : un parcours historique », in Y. coURBAGE \& M. KROPP (dir.), Penser l'Orient, Beyrouth, Institut français du Proche-Orient/Orient Institut ("Études contemporaines ») : 103-128.

2004 b - Orientales II. La III République et l'Islam, Paris, CNRS Éditions.

LECA, J.

1988 - « L'islam, l'État et la société en France. De la difficulté de construire un objet de recherche et d'argumentation ", Annuaire de l'Afrique du Nord, XXVII Paris, Éditions du CNRS.

LE PAUTREMAT, $P$.

2003 - La Politique musulmane de la France au Xxe siècle. De l'Hexagone aux terres d'Islam. Espoirs, réussites, échecs, Paris, Maisonneuve \& Larose.

LEVEAU, R. \& KEPEL, G. (DIR.)

1987 - Les musulmans dans la société française, Paris, Presses de Science Po.

LORCERIE, F.

1998 - « Berque, l'école, l'immigration : rencontre inopinée », in F. PouILLoN (dir.), Enquêtes dans la bibliographie de Jacques Berque. Parcours d'histoire sociale, Revue des mondes musulmans et de la Méditerranée, 83-84 : 171-194.

LUIZARD, P.-J. (DIR.)

2006 - Le choc colonial et l'islam. Les politiques religieuses des puissances coloniales en terres d'islam, Paris, La Découverte.

MARZOUKI, N.

2004 - « Théorie et engagement chez Edward Saïd », Mouvements, 33-34 (3) : 62-67.

MÉRAD, A

2007 - L’islam contemporain, Paris, PUF (« Que-sais-je ?», 2195) (8édition).

MOHSEN-FINAN, K. \& LEVEAU, R. (DIR.)

2005 - Musulmans de France et d'Europe, Paris, CNRS-Éditions (« Science Politique »).

SAÏD, E.

1980 - L'orientalisme. L'Orient créé par l'Occident, Paris, Éditions du Seuil.

SELLAM, S.

2006 - La France et ses musulmans. Un siècle de politique musulmane 1895-2005, Paris, Fayard.

TRIAUD, J.-L.

1998 - « L'islam vu par les historiens français », Esprit, 246 : 110-132.

DE VITRAY-MEYEROVITCH, E.

1995 - Islam. L'autre visage, Paris, Albin Michel (« Espaces libres »).

2005 - Rûmî et le soufisme, Paris, Éditions du Seuil (« Points Sagesse »).

WEIBEL, $\mathrm{N}$.

2000 - Par delà le voile : femmes d'islam en Europe, Bruxelles, Éditions Complexe. 


\section{NOTES}

1. Centre national de la recherche scientifique, École des hautes études en sciences sociales, École pratique des hautes études et Institut d'études politiques de Paris.

2. Sur l'histoire, le fonctionnement et la composition de ces différentes institutions, voir Pascal LE PAUTREMAT (2003).

3. Jacques Berque a entretenu une relation d'amitié avec Jean-Pierre Chevènement qui lui inspira un certain "orientalisme républicain", lorsque ce dernier fut ministre de l'Intérieur chargé des cultes et créateur du futur Conseil français du culte musulman (CFCM). À propos de l'influence de la pensée de Jacques Berque sur J.-P. Chevènement, voir Vincent GEISSER et Aziz ZEMOURI (2007).

4. Rémy Leveau (1932-2005) a développé ses premiers travaux sur le champ politique marocain, avant d'intégrer les cercles diplomatiques et de revenir à l'Institut d'études politiques de Paris pour former les nouvelles générations de politologues français sur le monde arabe et musulman et les questions d'islam européen (LEVEAU \& KEPEL 1987).

5. Bruno Étienne (1937-2009) a séjourné dans de nombreux pays du Maghreb avant d'initier à l'IEP et à l'Institut d'études et de recherches sur le monde arabe et musulman (IREMAM) d'Aix-en-Provence des recherches pionnières sur l'islam de France (ÉTIENNE 1989a, b, 1990).

6. L'intellectuel Gilbert ACHCAR (2008) se montre extrêmement sévère sur la posture scientifique adoptée par Gilles Kepel en le comparant à un «Bernard Henry Lévy de l'orientalisme français ». Cette critique nous semble largement exagérée car les travaux de G. Kepel ont toujours recouru à un appareillage méthodologique rigoureux, point soupçonnable de verser dans la vulgarisation philosophique.

7. On peut citer des sociologues comme Nelüfer GÖLE (1993), Françoise GASPARD (1995) ou Farhad KHOSROKHAVAR $(1997,2004)$ qui, par leurs recherches empiriques et théoriques, ont énormément apporté à la connaissance du fait musulman en France et en Europe.

8. On renverra le lecteur au site "Eurislam " qui met en ligne une base de données bibliographiques sur l'islam en Europe : <http://www.eurislam.info/>.

\section{RÉSUMÉS}

Résumé

L'émergence d'une "question musulmane » au sein de l'espace hexagonal a suscité un nombre croissant de travaux en sciences sociales depuis le début des années 1980. Contrairement à toute attente, ce ne sont pas les chercheurs arabisants issus des cursus d'islamologie classique qui ont investi ce nouvel objet mais davantage les sociologues et les politologues en phase avec les demandes sociales et politiques du moment. Ce désengagement de l'islamologie classique des problèmes musulmans $\mathrm{du}$ temps présent s'explique très largement par le processus de 
« décolonisation mentale » qui a touché les sciences sociales et le discrédit idéologique jeté sur la tradition orientaliste, contrainte de se replier sur le milieu universitaire et le champ académique, créant ainsi un vide dans la compréhension des phénomènes islamiques contemporains. À l'inverse, les politologues ont très largement investi l'objet «islam de France ", au risque de brouiller les frontières entre science et expertise.

The "Muslim Issue" in France Through the Prism of Social Sciences

Since the early 1980s, the emergence of a "Muslim issue" in France has led to a growing number of studies in social sciences. Contrary to expectations, this new subject of research was taken up more by sociologists and political scientists, attuned to contemporary social and political demands, than by Arabist researchers, who are the products of traditional Islamic studies. This disengagement of traditional Islamic scholarship from the present can largely be explained by the "mental decolonization" that has affected social science and the ideological disrepute that has been cast on the orientalist tradition, leading it to fall back on university research and academe, thereby creating a vacuum in the understanding of contemporary Islamic issues. Conversely, the political scientists have devoted themselves to "French Islam", at the risk of blurring the frontiers between science and expertise.

\section{INDEX}

Keywords : France, Decolonization, Expertise, Muslim Matters, Immigration, French Islam, Islamism, Muslim European, Orientalism, Political Science

Mots-clés : France, décolonisation, expertise, fait musulman, immigration, islam de France, islamisme, islamologie, islamophobie, musulmans européens, orientalisme, politologie

\section{AUTEUR}

\section{VINCENT GEISSER}

Institut français du Proche-Orient (IFPO-CNRS), Beyrouth ; Centre d'information et de documentation sur les migrations internationales (CIEMI), Paris. 